\title{
Du Gai Savoir de l'acteur à celui du spectateur : les paradoxes du rire selon Dario Fo
}

Anick Brillant-Annequin

\section{OpenEdition}

1 Journals

Édition électronique

URL : http://journals.openedition.org/recherchestravaux/288

DOI : 10.4000/recherchestravaux.288

ISSN : 1969-6434

Éditeur

UGA Éditions/Université Grenoble Alpes

Édition imprimée

Date de publication : 30 mai 2005

Pagination : 221-237

ISBN : 0151-1874

ISSN : 0151-1874

\section{Référence électronique}

Anick Brillant-Annequin, « Du Gai Savoir de l'acteur à celui du spectateur : les paradoxes du rire selon Dario Fo », Recherches \& Travaux [En ligne], 67 | 2005, mis en ligne le 27 janvier 2014, consulté le 08 septembre 2020. URL : http://journals.openedition.org/recherchestravaux/288 ; DOI : https://doi.org/ 10.4000/recherchestravaux.288 


\section{Du Gai Savoir de l'acteur à celui du spectateur : Les paradoxes du rire selon Dario Fo}

Dario Fo est de ces artistes complets, auteur, acteur et metteur en scène, qui associent l'invention du trouvère et l'agilité du jongleur dans une telle osmose qu'on ne sait plus s'il est jongleur de mots et de situations dramatiques ou trouvère de gestes, acrobaties et autres inventions corporelles et vocales. Un torrent d'énergie comique qui transforme les sujets ou problèmes les plus sérieux, voire sacrés, en fantaisies échevelées ou grotesques, qu'il s'agisse de l'«orifice sacré» du pape ou de la «résurrection de Lazare». La lecture de son Manuale minimo dell'attore apporte certes de nombreux conseils de jeu dramatique et délivre des connaissances précieuses pour les amateurs de théâtre et les acteurs, mais c'est avant tout un livre qui provoque à chaque page ou presque le rire du lecteur tant l'auteur le provoque, le sollicite de façon gaillarde, l'invite à envisager la réalité à travers une logique insolite et décapante qui ne peut que le réjouir dès lors qu’il a choisi de ne pas s'étouffer d'indignation.

C’est d'ailleurs pourquoi le titre français choisi par Valeria Tasca est Le Gai Savoir de l'acteur, qu' elle inscrit dans la tradition du gai saber des troubadours ${ }^{\text {I }}$ plutôt que dans la référence à Nietzsche. Mais il s'agit bien de lutter contre le préjugé qui associe le rire et la gaieté à l'absence de réflexion et à la dégradation de la pensée. À cette «bête sérieuse» (p. I4) Dario Fo va opposer sa vision darwinienne de l'évolution humaine qui commence avec l'homo faber, continue avec l'homo sapiens pour culminer avec l' homo ridens (p. 156) tant le rire est pour lui indissociable de l'intelligence et de l'esprit critique. Son Manuale minimo s'avère être en fait une somme de réflexions sur le rire et sur l'essence

I. P. I3 de l'avant-propos du Gai Savoir de l'acteur, D. Fo, trad. fr. V. Tasca, Paris, L'Arche, 1990. Dans la suite du texte, la pagination des citations de l'ouvrage sera référencée entre parenthèses immédiatement après celles-ci. 
du comique théâtral, et il nous invite à comprendre comment il est produit et quelles visées il poursuit.

Or, ses analyses, si drôles et apéritives, ne peuvent éluder la complexité de ce phénomène, qu'il l'inscrive dans une dimension socio-politique ou psychologique, car le rire peut s'exercer sur tout et tous, dominants haïs ou faibles persécutés, il peut être un outil de préservation ou de transformation sociale, il peut décaper la réalité ou l'euphoriser, il est par essence ambivalent. Mais il présente au moins une constante, son extraordinaire pouvoir de vivification: rire donne de l'énergie et le goût de vivre comme en témoigne le succès des diverses écoles du rire qui se sont créées ces dernières années pour diffuser les bienfaits de la ridothérapie.

C'est au mystère du rire que s'est consacré Dario Fo et les six conférences de ce manuel nous invitent à y participer en suivant son cheminement des origines à nos jours et en s'interrogeant sur le discrédit dont il est perpétuellement accablé et paradoxalement renforcé. La structure du rire semble précisément être paradoxale puisqu'il associe aussi bien des composantes épiques mettant en jeu la distance réflexive que l'effet cathartique qui dissout les angoisses, qu'il joue sur la réversibilité du comique et du tragique, et qu'il décerne ses traits aussi bien aux puissants qu'aux petites gens et aux inadaptés sociaux. Du coup, les effets psychologiques du rire dépendent certainement des dosages opérés par les artistes et surtout de la fonction sociale qui lui est impartie dans un champ culturel et une époque donnés. Que Dario Fo veuille en irriguer tous les lieux possibles et sous sa forme la plus dérangeante n'est pas pour étonner et montre assez que pour lui le rire est une arme politique et populaire particulièrement efficace.

\section{Un rire universel qui se nourrit des mythes et des rites ancestraux}

Le Gai Savoir de Dario Fo peut se lire comme un discours sur les mythes du théâtre, une mythologie du théâtre comique plus particulièrement, même si dans le mythe, discours des origines, une certaine indifférenciation générique semble être à l'œuvre. De ses origines rituelles, le théâtre conserve en effet l'usage du masque qui est l'une des composantes «constantes de toute religion archaïque» (p. 38) et Dario Fo remarque d'ailleurs une analogie troublante entre des masques indiens, chinois et japonais et certains masques de zanni comme Arlequin et Brighella: la présence d'une marque rouge ou d'une protubérance sur le front, qui serait la trace du troisième oil permettant la clairvoyance, propre aux magiciens, voyants ou êtres divins ou diaboliques (p. 42). Le théâtre hérite également d'une gestualité rituelle qui permet de transmettre un savoir et des compétences par le corps (p. 67). Ces deux éléments se retrouvent dans ces manifestations traditionnelles que sont 
les carnavals ou autres fêtes comme celles consacrées au printemps, les "Mais", ainsi que dans les passions ou les formes de théâtre religieux issues du Moyen Âge et encore jouées par des communautés villageoises ou urbaines. Le Gai Savoir conte, avec cette maitrise de l'hypotypose propre à son auteur, différentes manifestations rituelles et carnavalesques comme la sarabande des mammuttones en Sardaigne, les carnavals médiévaux où le double grotesque du gouverneur politique ou religieux de la cité parodiait ce dernier ou encore une passion jouée en Croatie. Celle-ci conterait un mythe très ancien où un cadavre fictif est hissé hors de sa tombe par les racines et les branches d'un arbre qui sont en fait représentées par les bras de la communauté à laquelle il appartenait. Ainsi le mort, en nourrissant l'arbre qui l'incorpore, participe encore au cycle de la vie et «il vit aussi, évidemment, dans l'esprit et dans le corps de tous ceux avec qui il a partagé affection, amitié, solidarité» (p. 264). On constate que Dario Fo fait référence à des mythes et rites qui privilégient une vision résolument positive de la vie et transgressive de l'ordre social, «un théâtre qui sait affronter avec ironie jusqu'aux lois divines» (p. 265).

Cette préférence pour les mythes de survie et de renversement de l'ordre social et naturel trouve sa plus complète expression dans ce que Franca Rame, la compagne de Dario Fo, présente comme l'origine mythique du comique, ce genre étant d'ailleurs présenté comme précédant et générant le tragique. En effet, la nourrice Baubô, pour divertir Déméter de son désespoir dû à l'enlèvement de sa fille Coré par Hadès, se dénude, maquille son corps en un grand visage et exécute des danses et des chants comportant des passages obscènes, ce qui fait rire la déesse et permet ainsi le « retour de la joie et de la vie dans la création [...] dans le monde des hommes» (p. 292). Or cette scène bouffonne serait à l'origine du «rite d'Éleusis, forme première du spectacle tragique» (ibid.).

Cette puissance du rire qui redonne la vie n'est pas propre à la civilisation occidentale puisqu'on retrouve un mythe sensiblement analogue à l'origine du nô japonais :

Dans les deux plus anciennes formes de tragédie qu'on connaisse, nous avons donc au départ la catharsis par le rire et l'obscénité sexuelle qui libèrent la lumière et l'harmonie. (p. 294)

D’ailleurs, Dario Fo insiste sur les analogies et filiations entre théâtre oriental et occidental. C'est ainsi qu'il souligne la parenté entre certains masques de la commedia dell'arte et ceux du théâtre grec qui eux-mêmes puiseraient dans les masques orientaux, dévoilant de ce fait "l'itinéraire des migrations culturelles entre l'Orient et la Méditerranée, du monde archaïque à celui de la commedia dell'arte» (p. 40). Il établit également des parallèles 
entre des formes anciennes de mystification et provocation théâtrales qu'on trouve aussi bien en Inde, dans les spectacles médiévaux et des comédies en Occident que dans les formes plus récentes du happening et du «théâtre de l'invisible" pratiqué par Augusto Boal2. C'est dans ce grand courant comique qui irrigue les arts de la représentation scénique depuis l'Antiquité que Dario Fo conseille de puiser car selon lui « rien ne suggère mieux des images d'avantgarde que l'observation de nos fêtes de carnaval» (p. 159).

Certaines techniques du comique, comme l'exploitation d'un incident provoqué ou non et l'improvisation, unissent ainsi le théâtre des jongleurs, la commedia dell'arte et l'art du clown qui lui-même puise à son tour dans les farces antiques. Du coup, Dario Fo propose son propre mythe de la naissance de la commedia dell'arte née "d'un mariage obscène entre "jongleresses", conteurs des rues et clowns, puis, à la suite d'un inceste, la commedia a accouché de dizaines d'autres clowns» (p. 24I).

\section{La recette du rire selon le Manuale minimo}

Dès lors, on peut aisément identifier certains ingrédients indispensables à la production du rire dans le cadre du spectacle comique. L’obscénité, comme le montre la citation précédente, et son associée la scatologie, en sont des composantes majeures. Ainsi les premiers Arlequin comme Martinelli puis Biancolelli usaient de mots et gestes obscènes très provocateurs comme le fait de déféquer à l'avant-scène et de projeter sur le public le prétendu résultat de leurs efforts (il s'agissait heureusement d'un substitut de châtaignes cuites!). Les clowns ou bouffons des origines étaient ainsi assimilés à des "catapultes obscènes» selon Dario Fo (p. 240) qui bien entendu, honore la tradition avec délectation en dissertant, par exemple, sur «l'orifice sacré» du pape dans le contexte au demeurant fort dramatique de l'attentat dont ce dernier avait été victime.

L'obscénité, liée aux manifestations les plus triviales du corps, présente selon Dario Fo un pouvoir de libération des plus efficaces. Elle permet de lutter contre "le sentiment de la faute, la honte et l'angoisse du péché» et de «combattre cette angoisse par le rire» (p. 277). L'obscène apparaît donc comme une force, un contre-pouvoir que Dario Fo illustre entre autres en citant un témoignage historique rapporté par une spécialiste du théâtre médiéval allemand, Katrin Kröll: lors d'une bataille opposant au XIV siècle l'armée française constituée de professionnels et l'armée bâloise composée essentiellement de volontaires non aguerris, des jongleurs se déploient devant

2. A. Boal, Jeux pour acteurs et non-acteurs, pratique du théâtre de l'opprimé, Paris, La Découverte, 1983 . 
les Bâlois et provoquent les Français qui restent impassibles jusqu'au moment où les bouffons

[...] baissent culotte, font éclater des pétards comme s’ils leur déféquaient à la figure et se nettoient le derrière avec des drapeaux qui reproduisent ceux des Burgondes,

ce qui provoque la réaction de ces derniers, partant à la poursuite des pitres, en dépit des ordres de leurs chefs leur intimant de revenir sur leurs lignes. Or, c'est bien ce que visaient les Bâlois qui, avantagés en matinée par leur position par rapport au soleil, voulaient avancer le début de la bataille pour profiter de cette supériorité stratégique qui leur procura la victoire. Force incontestable de l'obscénité qui vient ainsi au service des militaires les moins experts et des plus faibles (p. I26)!

On le constate, le corps porte et suscite le rire, pas seulement le «bas» du corps, particulièrement sollicité dans l'obscénité et la scatologie, mais aussi l'ensemble des capacités corporelles privilégiées dans la commedia dell'arte dès lors qu'elle est dans l'obligation de quitter l'Italie et que les comédiens italiens doivent se faire entendre par des étrangers dont ils ne maîtrisent pas la langue. Contraintes que l'on retrouve d'ailleurs chez les clowns contemporains de dimension internationale qui sont amenés à jouer dans divers pays. Dario Fo souligne ainsi «la nécessité de développer l'intelligence du geste et l'agilité du corps" (p. 97), qui donne naissance, notamment, aux effets comiques des lazzis ou des sketches. Le rire semble donc privilégier le langage non verbal du corps.

Allant plus loin, divers traits associent l'effet comique à la déshumanisation de l'expression non verbale. Ainsi, Dario Fo analyse longuement l'aspect zoomorphe des masques de la commedia évoquant la basse-cour, les volailles, chats, chiens et porcs mais aussi les singes (p. 39) qui vont de pair avec la posture et la gestuelle des animaux concernés: Pantalon, par exemple, meut son corps comme un dindon. La transformation végétale peut être également une composante, particulièrement dans les formes primitives de l'Arlequin, couvert de feuilles multicolores, que Dario Fo situe en France dans la tradition de l'homo selvaticus carnavalesque (p. 80). Poussant encore plus loin la déshumanisation, on atteint la mécanisation: le corps humain de l'acteur se muant en une marionnette, mutation favorisée par la présence de masques. Ainsi, des modes de déplacement et de mouvement des membres et du buste de l'acteur sont analogues à ceux des marionnettes: "dislocation, sautillements, soubresauts, moulinets des bras, torsion brusque du buste, du cou et de la mâchoire, d'un comique irrésistible» (p. 44).

Ce rire né de la métamorphose de l'humain en animal, végétal ou machine, sent la magie sinon le soufre, et c'est fort logiquement qu'il entre en accointance 
avec le diable. On a déjà signalé cette protubérance, vestige du surnaturel ou du diabolique, sur certains masques orientaux et celui du zanni. Le masque d'Arlequin serait ainsi le «croisement du zanni bergamasque avec les personnages diaboliques des farces françaises de tradition populaire» (p. 78), Dario Fo partageant d'ailleurs la croyance en l'origine démoniaque du nom même d'Arlequin issu du Helleken ou Hellequin médiéval, personnage diabolique et farcesque.

\section{Une recette qui n'est pas au goût de tous}

Tous ces ingrédients, qui concourent à la création d'une forme très populaire de théâtre, sont autant de facteurs de dénigrement et de dégoût de la part des doctes. Les genres qui provoquent le rire sont traditionnellement considérés comme inférieurs, ce sont des formes dégradées du théâtre si l'on en croit la classification héritée d'Aristote, et qui perdure même dans les représentations culturelles contemporaines. Or Dario Fo refuse «ce schématisme imbécile»:

Refuser les définitions, les jugements de valeur, les classifications de type aristotélicien - je veux dire: dans l'échelle des valeurs il y a d'abord la tragédie, puis le drame, puis la comédie, et ainsi de suite, jusqu'au théâtre de marionnettes, au saltimbanque, au paillasse. (p. I23)

Il déplore ainsi la suppression du personnage comique dans les versions actuelles de certaines fêtes populaires comme les «Mais» qui du coup perdent leur contrepoint ironique aux discours nobles et sérieux, et il est particulièrement acide avec «les intellectuels agnostico-littéraires» qui ont «dédaigné les clowns, les saltimbanques, les marionnettes» (p. 153).

Ces formes d'art populaire pâtissent également du discrédit dont souffre le théâtre sans texte. Ainsi le théâtre de Guignol tout comme la commedia dell'arte ne feraient pas partie de l'art puisqu'ils existent indépendamment de l'écrit. Dario Fo critique à cet égard le jugement esthétique de Diderot pour qui le texte est prééminent, et qui est emblématique des attitudes des lettrés européens. Et comme dans les formes de théâtre sans texte, le comique occupe une place primordiale, celles-ci souffrent d'une double déconsidération puisque le rire est posé comme antagoniste à la raison qui, selon ces mêmes doctes, ne peut s'exprimer que par la pensée sérieuse.

Il n'est guère étonnant que ce théâtre, qui vit de mystifications, de provocations plus ou moins improvisées à l'égard des valeurs consacrées, soit considéré par les institutions dominantes et ses représentants comme une dégradation, voire une négation, de l'art dramatique. Et cependant, il n'a cessé d'exister et de traîner à sa suite un public hilare tant la force du rire l'emporte en définitive sur toute censure ou jugement de goût. Mais sa puissance réside peut-être avant tout dans sa structure paradoxale qui entraîne une réception du même ordre de la part des spectateurs. 


\section{Le premier paradoxe du rire: la combinaison de l'épique et de l'effet cathartique}

Dans Le Manuale minimo le rire apparaît comme l'un des meilleurs outils pour développer le sens critique en usant de l'effet de distanciation qu'il permet de créer et en sollicitant une réception rationnelle de la part du spectateur. Mais en même temps, en délivrant ce dernier de ses tensions, il agit directement à des niveaux non conscients par son pouvoir cathartique. Combinaison donc ou plutôt mécanisme dialectique entre ces deux aspects du rire.

Dario Fo décrit et illustre constamment le jeu épique de l'acteur. Ainsi dans son manuel, il décrit minutieusement sa technique de comédien pour la jonglerie de La Résurrection de Lazare où un seul acteur joue une foule de personnages avec des démultiplications extrêmement rapides et des adresses au public: on est résolument dans une forme de théâtre épique que l'acteur développe jusqu'à impliquer "physiquement le public, jusqu’à le transformer en un chœur dont tous les membres sont présents à côté de moi sur la scène» (p. I45). Il s'agit évidemment d'un déplacement symbolique signifiant le rôle actif que Dario Fo exige de son public, public qui «doit toujours être présent, conscient de son rôle»(ibid.). L'esthétique de ce jeu est caractérisée par l'importance de l'ellipse et de la synthèse, la suppression "de tout superflu, toute description ennuyeuse» (p. I48), le refus affirmé du réalisme. Allusif et condensé, le spectacle sollicite constamment l'attention du spectateur sans l'ennuyer, selon «la grande tradition épique populaire» (ibid.).

Et bien sûr le comique n'est pas pour peu dans l'intérêt du spectateur et dans l'esthétique épique des spectacles de Dario Fo. En effet,

[...] l'éclat de rire révèle le sens critique, la fantaisie, l'intelligence, le refus de tout fanatisme.[...] Quand il s'exprime, le petit peuple (il popolo minuto), les gens simples ne peuvent s'empêcher, même dans les histoires tragiques, d'introduire l'humour, le sarcasme, le paradoxe comique [...] (p. 156)

Le comique crée ainsi une distance critique, il instaure dans les situations dramatiques ou tragiques, "sérieuses", un contrepoint qui propose une vision dialectisée de la réalité et qui fait appel au sens critique du public.

Dario Fo assigne clairement au théâtre une tâche civique et politique:

C'est notre devoir, ou si vous préférez notre tâche professionnelle d'auteurs, metteurs en scène, gens de théâtre, de parler de la réalité en brisant les schémas attendus, par l'imagination, l'ironie, le cynisme de la raison. (p. 164)

On est bien dans la tradition de l'effet de distanciation brechtien, mais la spécificité de Dario Fo est de s'appuyer résolument sur le comique pour le 
susciter. Et d'ailleurs, il ne cesse de rappeler la tradition très ancienne du jeu épique lui-même qu'il fait remonter aux origines du théâtre grec où l'acteur devait proscrire l'identification aux personnages, et qui traverse les formes du théâtre populaire.

Dans l'économie d'un spectacle comique, les incidents, provoqués ou non, et les improvisations des acteurs, éveillent le public en le mettant au cœur de l'action dramatique:

[...] l'incident ne sert pas seulement à ranimer des schémas usés, mais à rompre un autre schéma mortifere, celui qui réduit le spectateur au rôle de voyeur [...] (p. IIo)

L'incident vivifie le stéréotype en rendant le spectateur actif, en brisant ce quatrième mur que les dramaturges et acteurs comiques ne cessent de franchir. Dario Fo cite ainsi de nombreux exemples, dont celui de la guêpe qui, de désagrément très réel, s'est transformé en lazzi comique et même en comédie. Il illustre également son propos de l'exemple de Molière qui privilégiait le jeu en avant-scène, projetant en quelque sorte les acteurs au milieu des spectateurs.

Mais le malin génie comique peut pousser encore plus loin la participation du public, en usant de la mystification et de la provocation. On en trouve des exemples aussi bien dans les spectacles médiévaux, les comédies italiennes des $\mathrm{XVI}^{e}$ et XVII ${ }^{e}$ siècles, les spectacles indiens et iraniens, ce qui atteste de l'universalité du phénomène, ainsi que dans les formes actuelles du "théâtre de l'invisible" développé par Augusto Boal ${ }^{3}$ ou dans des propositions faites par la troupe de Dario Fo et Franca Rame. Dans tous les cas, il s'agit pour les comédiens de s'immerger de façon anonyme dans un groupe et de jouer des situations problématiques qui vont provoquer chez le public des réactions fortes et contradictoires. Ainsi en Inde, il s'agira d'une mystification qui met en scène une femme à laquelle son mari est censé avoir coupé le nez pour punir son infidélité, ce qui correspond à une coutume encore en usage dans certaines régions du pays. Cela entraîne des réactions opposant les «modernistes» critiquant cette pratique inhumaine aux «réactionnaires» qui insultent la femme infidèle. Celle-ci pousse alors le jeu jusqu'à les accuser d'avoir été ses amants, ce qui entraîne des réactions en chaîne de plus en plus violentes jusqu'au moment de la démystification où les spectateurs piégés font l'objet des moqueries les mieux senties. Dario Fo cite également l'une de ces ruses dramatiques créée à l'occasion d'un de ses spectacles, Guerra di popolo in Cile, au cours duquel les acteurs font croire à la réalité d'un coup d'État en Italie, se déroulant au moment du spectacle et entraînant l'arrestation pour

3. Voir note précédente. 
«vérification d'identité» de plusieurs membres du public. Les réactions des spectateurs révèlent alors leur attitude face à ce coup de force et leurs convictions profondes, ce qui entraîne après la démystification des réactions violentes et beaucoup de rires et sarcasmes à l'encontre de ceux qui avaient révélé leur lâcheté, voire leur propension à la collaboration avec les dominants. Ce "procédé de camelot", selon les critiques des esthètes indignés, comporte en fait selon Dario Fo une «signification morale» (p. 276) puisque cela permet, en masquant la donne théâtrale, de démasquer les hypocrisies et d'éveiller la conscience. Mais le sociodrame ainsi suscité, en se recontextualisant dans le domaine esthétique du théâtre, provoque un rire d'autant plus fort qu'il soulage les intéressés et qu'une partie de la communauté peut s'amuser aux dépens de celle qui porte les traditions rétrogrades faisant obstacle à l'émancipation humaine. On note que ces pièges théâtraux mettent toujours en scène des situations graves, voire dramatiques, où les enjeux portent sur des biens et des valeurs essentiels, ce qui permet des révélations, pour soi et pour l'autre, d'autant plus efficaces et engendre une réaction par le rire d'autant plus intense.

Mais on perçoit bien que ce rire qui soulage parce qu'il correspond au relâchement d'une forte tension, se situe aussi du côté de la catharsis. Franca Rame, en faisant référence aux origines de la tragédie grecque et au nô japonais, rappelle que c'est

[...] au départ la catharsis par le rire et l'obscénité sexuelle qui libèrent la lumière et l'harmonie. Colère, haine, peur sont ainsi, dans toutes les représentations populaires, exorcisées et résolues dans le jeu grotesque [...] (p. 294)

On a vu que même la mort peut faire l'objet de ce rire libérateur.

Le jeu mécanisé de certains acteurs comiques, calqué sur la posture et la gestuelle des marionnettes, participe également à la création d'une tension dont nous délivre le rire. Sigmund Freud a montré dans « L'Inquiétante Étrangeté » 4 à quel point le «thème de la poupée animée» (p. I84), objet d'un désir infantile, devient un objet d'angoisse à l'âge adulte. Il s'appuie sur les analyses du «double» développées par Otto Rank dans Une étude sur le doubles pour montrer que ce qui, au départ, était une forme de survie par-delà la mort, devient, par inversion, "un étrangement inquiétant signe avant-coureur de la mort» (p. I86). L'acteur-marionnette, jouant de l'ambiguïté entre vivant et inanimé, suscite ainsi un malaise, une angoisse que le rire là encore exorcise.

4. S. Freud, "L'inquiétante étrangeté» dans les Essais de psychanalyse appliquée, trad. M. Bonaparte et E. Marty, Paris, Gallimard, 1976, Ie éd. fr. 1933.

5. O. Rank, Une étude sur le double, Paris, Denoël et Steele, I932. 
L'alternance entre tension et relâchement, nécessaire à l'effet comique et au rire, suppose une maitrise du rythme du spectacle. Lorsque Dario Fo explique son jeu dans La Résurrection de Lazare, il insiste sur ce point, sur la nécessité des "pauses", notamment après la décharge énergétique du rire:

[...] il faut faire respirer le public avec soi, le public doit lui aussi pouvoir se reprendre. Si on l'essouffle, si on ne le laisse pas respirer après les moments de tension, ou à la fin d'un éclat de rire, si on l'agresse sans cesse, on finit par le fatiguer, il ne peut plus participer comme il faut et il ne s'amuse plus. (p. I50)

À l'instar de Ionesco, il compare volontiers le spectacle de théâtre à un «combat sans ring où les coups de poing alternent avec les caresses» (p. I6I), soulignant ainsi l'importance de l'impact émotionnel sur le spectateur.

Ainsi, l'acteur comme le spectateur doit pouvoir éprouver des émotions tout en conservant sa lucidité. Dario Fo évoque un savant dosage entre "l'émotif et le rationnel, un équilibre dynamique et non statique» que les comédiens dell'arte notamment savent maintenir (p. 30), permettant ainsi une participation du public combinant la distance et la connivence. Il pose ainsi le problème du jeu, du passage entre conscient et préconscient chez l'acteur, d'une certaine élasticité psychique qui autorise ce dernier à franchir le seuil sans risque ni maladresse pour lui ou pour son public.

Ce qui favorise une telle qualité de jeu est le recours, qu'il s'agisse de développer l'esprit critique ou d'obtenir un effet cathartique, à l'imaginaire de l'acteur et du spectateur. Dario Fo, pour qui «le théâtre est fiction et non imitation" (p. 202), privilégie un jeu qui fait appel à l'activité symbolique. Ainsi, tout ce qui relève des figures de déplacement et de condensation sera mis en œuvre pour produire un art allusif, à l'opposé d'un art descriptif qui ne sait pas «focaliser sur certains détails et glisser sur d'autres» (p. 182). Poussant ainsi le raisonnement jusqu'au paradoxe, un de plus, il affirmera que "au théâtre, seul le faux est authentiquement vrai» (p. 250).

L'utilisation du masque, notamment, favorise ces processus de symbolisation puisque le port de ce dernier, en privant l'acteur de l'expressivité du visage, déplace et condense le jeu sur le corps. Celui-ci devient le «cadre» du masque qui ne prend sens qu'en fonction de la gestuelle et de la posture de l'acteur. Celles-ci doivent d'ailleurs être accentuées et développées, le masque imposant la justesse et l'élargissement du geste, surtout si un effet comique est recherché (p. 63). Dario Fo remarque, à l'instar de Peter Brook et de Giorgio Strehler, que le port du masque, après un temps d'adaptation, libère l'acteur et lui permet, paradoxalement, d'être soi. Ainsi Giorgio Strehler explique qu'après bien des réticences, le grand Arlequin Marcello Moretti avait accepté de jouer avec le masque. Il avait ainsi découvert l'importance expressive de la bouche dans le demi-masque de la commedia dell'arte. Mais 
surtout, le masque avait permis à cet acteur pudique de se libérer, de «faire affluer toute une vitalité, une fantaisie non "réaliste" mais toutefois ancrée dans sa nature populaire intérieure». Grâce à cette contrainte, il «représenta ainsi la partie la plus vivante de lui-même» ${ }^{6}$. Le masque oblige à "dire la vérité», renchérit Dario Fo, car il gomme le visage, lieu du faux-semblant, au profit du corps, or «personne n'est habitué à mentir avec son corps. Étienne Decroux disait: "Si tu sais observer et déchiffrer le langage du corps, rien ne t'échappera des mensonges d'autrui”" (p. 66).

Le mime sollicite également énormément l'imaginaire de l'acteur et de son public, en recourant aux mêmes processus figuratifs, l'allusion, l'ellipse, l'amplification et la décomposition gestuelles. Le mime signifie même un objet et un décor absents, poussant l'art du déplacement et de la condensation à l'extrême, il est le grand «manipulateur» de la réalité qu'il recrée en transformant son corps en "cette usine de rêves" prônée par Vitez 7 à propos de sa classe de Conservatoire. Dario Fo se réfere constamment au jeu masqué et au mime même lorsqu'il joue en usant de la parole à visage découvert. L'exemple de son jeu dans La Résurrection de Lazare est significatif à cet égard puisqu'il procède constamment par resserrement, condensation, suggestion, sollicitant ainsi perpétuellement un travail de complétude du sens de la part du spectateur. C'est grâce à cette puissance de synthèse que l'imaginaire s'épanouit et que le rythme du spectacle gagne en efficacité comique. Dario Fo cite ainsi en exemple l'élaboration de l'Histoire du tigre dont il ne cesse de resserrer le texte pendant une quinzaine de jours et qui, paradoxalement, passe de 25 à 55 minutes de représentation. Mais c'est bien là la clé du rire:

[...] au théâtre, souvent, quand on supprime des mots, le tempo s'élargit, parce qu'on fait une place aux pauses, aux rires, à l'amusement de l'acteur et du public. Par curiosité, j'ai voulu mesurer la durée des rires et des applaudissements dans l'enregistrement de la première représentation $[. .$.$] trois minutes et demie en$ tout. Et dans la dernière soirée, dix-huit minutes sur cinquante-cinq. Voilà où est la clé du mystère. (p. I88)

Mais il faut aussi, "pour garder le rythme, absolument», interrompre les applaudissements et les rires et continuer le jeu (p. 186). Resserrer donc, toujours, en tranchant dans l'effet même qui est recherché, le rire.

\section{Le second paradoxe: réversibilité du tragique et du comique}

Il est d'usage d'opposer le registre tragique au comique, chacun correspondant à une vision du monde et une posture en quelque sorte anthropologique

6. G. Strehler, Un théâtre pour la vie, trad. E. Genevois, Paris, Fayard, I980, p. I68-I69.

7. A. Vitez, Écrits sur le théâtre, I, L'École, Paris, POL, 1994, p. I38. 
face au réel. Le Gai Savoir propose au contraire une vision particulière et paradoxale fondée sur la réversibilité du comique et du tragique.

Franca Rame a ainsi souligné que dans leur forme archaïque, les «tragédies se développaient à partir de schémas comiques et même bouffons» (p. 29I). Dario Fo montre également comment la situation transforme le genre ou le registre en s'appuyant sur des exemples de jeu très concrets: il propose à trois acteurs de jouer la même séquence de mime. Pendant que ceux-ci sont en coulisse, il explique aux spectateurs les trois situations dramatiques différentes que celles-ci vont signifier et qui vont provoquer des colorations dramatiques ou comiques selon le cas:

Il est donc bien évident que la situation transforme la signification des gestes du pathétique au tragique, de l'humoristique au grotesque et même à l'obscène. Trois mimes identiques, trois résultats dramatiques différents. (p. 137)

On peut supposer qu'une telle polyvalence générique est favorisée par l'ouverture signifiante du mime qui repose sur le langage non verbal. Mais il suffit de comparer certaines scènes de Molière et de Racine pour s'apercevoir que la structure dramatique et les relations des personnages sont identiques. Il en est ainsi dans les scènes où un tiers caché influence le discours que l'un des personnages doit tenir à un interlocuteur qui ignore tout de la situation (scène 5 de l'acte IV de Tartuffe et scène 6 de l'acte II de Britannicus, par exemple) ou encore dans les scènes de séduction où un homme amoureux et dominant tente de séduire en vain une femme qu'il tient en son pouvoir mais qui repousse ses avances (scène 4 de l'acte $V$ de L'École des femmes et scène 4 de l'acte I d'Andromaque, où la différence de statut et de pouvoir de nuisance de l'homme et la différence d'enjeu - le couvent pour Agnès et la mort de son fils pour Andromaque - inclinent l'une vers le comique, l'autre vers le tragique).

Sur un autre plan, celui du jeu, Dario Fo établit des passerelles entre les deux registres. C'est ainsi que la maitrise technique considérable que doit acquérir un clown, tant au niveau de la voix, du jeu corporel, de la musique, du jonglage, de l'acrobatie, entre autres, serait des plus utiles dans les rôles tragiques.

Outre la réversibilité, tragique et comique, ou sérieux et comique entrent en résonance, grâce à certains personnages comme le "fou ", le «sot», présents dans des formes de théâtre "sérieuses» depuis le Moyen Âge. En plus d'un contrepoint comique, ce personnage permet, à l'instar du fool dans le Roi Lear, «le renversement constant du jeu » et impose « une dialectique à l'histoire et aux personnages» (p. I55). Cette coexistence des registres et des personnages opposés provoque un constant décentrement qui rejoint les deux composantes paradoxales du rire développées précédemment, la distanciation critique et l'adhésion émotionnelle. Dario Fo y voit là une forme d'expression éminemment 
populaire puisque «quand il s'exprime, le petit peuple (il popolo minuto), les gens simples ne peuvent s'empêcher, même dans les histoires tragiques, d'introduire l'humour, le sarcasme, le paradoxe comique» (p. 156).

En fait, ce processus de réversibilité ou de coexistence, selon les cas, révèle les conditions d'émergence du comique: c'est parce qu'il y a une condition humaine tragique, un "fond de désespoir, de peur et de colère", que va se «mettre en scène la dérision, le paradoxe et le ricanement» (p. 158), formes du rire particulièrement critiques, voire cyniques.

\section{Le troisième paradoxe: le rire comme contre-pouvoir et comme outil de domination}

Toutefois, si le rire apparait ainsi comme une forme de contre-pouvoir, au service du petit peuple, il peut être aussi un outil puissant de domination sur les éléments les plus méprisés d'une société donnée, remplissant ainsi des fonctions idéologiques et politiques contradictoires.

Certes, la fonction critique et contestataire du rire est l'une des plus constamment signalées et Dario Fo s'inscrit là dans une tradition des plus anciennement pratiquées et analysées. C'est parce qu'il permet la satire des dominants et de leurs valeurs, comme c'est le cas dans les rites carnavalesques, qu'il met à nu les processus de leur domination, de la violence symbolique ou réelle qu'ils imposent, qu'il dévoile l'hypocrisie à l'œuvre derrière tout cela, que le rire apparaît à Dario Fo comme l'outil de lutte le plus efficace contre les pouvoirs en place. Pour ce faire, il reprend la grande tradition des jongleurs qui prône «l'ambiguïté, la contradiction de toutes les valeurs établies» (p. I24), notamment dans Mystère bouffe ${ }^{8}$, dans lequel la reprise parodique des mystères permet un renversement désopilant des mythes les plus sacrés. Mais si le rire s'attaque notamment aux miracles du Christ, à sa Passion, si l'ironie s'exerce avant tout sur «la propre crédulité du peuple, sa naïveté, (elle) procède d'une tendresse complice, d'un esprit de connivence; elle réveille la conscience et suscite la solidarité9». Se moquer des puissants, c'est d'abord se moquer de son propre manque de clairvoyance, comme le dit le roi David à ses sujets:

Peuple stupide et misérable et aussi un peu couillon, mais pourquoi tu crois à toutes ces histoires? ${ }^{10}$

8. D. Fo, Mystère bouffe, jonglerie populaire, adaptation française G. Héry, D. Bonetti, A. Gauthier et C. Perrus, Paris, Dramaturgie Éditions, 1984, I $^{\mathrm{re}}$ éd. Mistero buffo, G. Bertani editore-Verona Nuova edizione, 1973.

9. J. Guinot et F. Ribes, "Le métier d'acteur, paradoxe du théâtre politique», Le Théâtre d'intervention depuis 1968, Lausanne, L'Âge d'homme, 1983, repris dans Mystère bouffe, op. cit., p. I89-190.

Io. Mistero buffo, op. cit., p. 49, texte traduit. 
Dans le même esprit, des clowns indiens ont coutume de se mêler aux fêtes religieuses et de se déguiser en saints hommes qui se livrent à des actes de plus en plus irrévérencieux, tournant ainsi en dérision les rites religieux, provoquant selon le public rires ou indignation (p. 252).

Cette démythification faite par des jongleurs et des clowns bouscule et secoue mais, grâce au rire, elle permet de dépasser le traumatisme que provoque toute désillusion et prise de conscience et de refuser l'apitoiement pour faire place à une attitude plus dynamique.

En s'appuyant sur les travaux de Katrin Kröll déjà citée, Dario Fo montre que les jongleurs en Allemagne et en Europe centrale, pouvaient même exercer une critique très directe et virulente des pouvoirs économiques, politiques et religieux et jouer le rôle d'agents de liaison et de propagandistes, comme ce fut le cas dans les guerres de paysans du XIV e au XVII ${ }^{e}$ siècle. La commedia dell'arte, après avoir poursuivi des buts analogues à ceux des jongleurs en Italie et en avoir été chassée pour cela même par la Contre-Réforme, a trouvé refuge en Europe, notamment en France et à la cour d'Henri III où l'Arlequin Tristano Martinelli, très apprécié par le roi lui-même, pouvait se livrer à «des satires appuyées contre des hommes politiques, des prélats et des nobles, assuré qu'il était de l'impunité» (p. 82). Dario Fo souligne d'ailleurs l'influence très positive qu'exerça la contrainte imposée par la Contre-Réforme italienne sur l'évolution de la commedia qui dut, de la fin du XVIe à la fin du $\mathrm{XVII}^{\mathrm{e}}$ siècle en France puis dans toute l'Europe, inventer de nouvelles formes de jeu mimique et acrobatique, une «langue» non identifiable mais hyperexpressive, et jouer en virtuose des structures dramatiques complexes proposées par les canevas. Ainsi, au lieu de circonscrire l'influence de la commedia dans un seul pays, la censure catholique italienne lui permit de s'exercer largement ailleurs et d'inspirer des auteurs comme Molière et certainement des Élisabéthains, notamment Shakespeare.

Ainsi, le rire, permettant de rétablir un ordre plus juste, est perçu comme une arme propre au peuple. Il s'appuie sur des valeurs laïques et développe des points de vue ironiques mettant à distance critique les valeurs consacrées par la hiérarchie sociale, la religion et la morale officielle. Il peut être aussi le résultat des mystifications auxquelles un public abusé participe, mettant ainsi en évidence les conflits traversant une communauté.

Mais par cela même, il peut aussi se retourner contre les acteurs de ce rire iconoclaste. On a remarqué que l'Arlequin Martinelli pouvait exercer sa virulence grâce au soutien du roi et que la verve critique de Molière avait grand besoin du soutien de Louis XIV. Mais lorsque ce soutien fait défaut, auteurs et comédiens sont en danger. C'est ainsi que les comédiens italiens de la commedia, après avoir été chassés d'Italie, le furent de France vers I695 pour les 
mêmes raisons d'ordre moral et politique. Dario Fo considère aussi que Molière remplissait la fonction de "bouc émissaire» lorsque Louis XIV devait détourner de lui les tensions morales et idéologiques (p. 98), comme on l'a vu à l'occasion de Tartuffe et de Don Juan, notamment. Auparavant, les habiles jongleurs qui parodiaient les seigneurs politiques ou religieux de la cité lors du carnaval devaient être sur leurs gardes, arriver masqués et s'éclipser promptement après le spectacle sous peine d'être rossés par les représentants de l'«ordre» (p. 257). En Angleterre, dans la période élisabéthaine, à la suite de la représentation du Chevalier au pilon flamboyant de Beaumont et Fletcher, parodie de drame merveilleux qui fait une satire sanglante des riches commerçants londoniens, le succès comique fut tel que «le théâtre a été brûlé par les marchands de la city» (p. 254).

C'est pourquoi le pouvoir a toujours tenté d'entraver ce rire libre en lui imposant chicanes bureaucratiques et économiques et, de façon plus franche et récurrente, l'instrument de la censure. Dario Fo cite plusieurs exemples

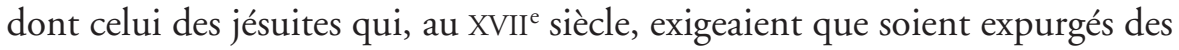
productions dramatiques les personnages démoniaques, ivrognes et d'une façon générale, indécents (p. 156). Au XXI siècle encore on peut déplorer que la censure, sous la forme du "politiquement correct» ou par le truchement des innombrables groupes de défense de l'image et des intérêts de collectivités spécifiques, s'exerce avec une efficacité certaine sur les jongleurs contemporains de la scène, particulièrement lorsque celle-ci est télévisuelle ou radiophonique.

Mais le rire n'est pas uniquement au service des groupes sociaux dominés. Il est même très fréquemment un instrument efficace de domination. On n'évoquera pas celui qui, dénué de toute référence socio-politique, morale ou idéologique, joue des stéréotypes et est, par sa vacuité même mais aussi par son pouvoir euphorisant qui demeure malgré tout, une remarquable forme de diversion et de manipulation. On abordera plutôt celui qui repose sur la moquerie à l'égard des "petits", des classes intermédiaires ou encore des individus ou groupes sociaux incapables de s'adapter à l'évolution historique.

On a déjà rappelé que la majorité des masques de la commedia s'inspirent des animaux de «basse-cour»: coq, dindon ou poule pour Pantalon, chien pour le Capitan, porc pour le Docteur, chat pour Arlequin (ou singe, ce qui échappe à la catégorie en question, tout en confirmant l'aspect zoomorphe). Les postures et modes de déplacement sont à l'avenant et évoquent une cour de ferme. En revanche, les nobles ne portent jamais de masque et conservent leur aspect humain. La partition sociale est ici évidente: la bourgeoisie moyenne représentée par les médecins et les marchands, les classes populaires représentées par les zanni, font l'objet de la charge comique et critique. La "haute-cour", quant à elle, constituée par 
[...] la haute noblesse, les grands négociants et les banquiers, on ne s'y frottait pas, par crainte de se voir rompre les os et chasser de la ville. (p. 40)

En fait, c'est «la bourgeoisie capitaliste naissante qui, en ce temps-là, gérait toute la culture, y compris le théâtre» (ibid.) et imposait les thèmes ainsi que la sélection des personnages à critiquer. Ceux-ci sont en fait caractérisés par leur inadaptation à l'évolution de la société: scolastique ridicule du Docteur, pratique de l'usure contraire à l'essor capitaliste et à l'esprit d'entreprise de la part de Pantalon par exemple. On notera des caractéristiques analogues dans les textes de Molière se moquant des bourgeois inadaptés à l'évolution de leur condition sociale comme M. Jourdain, qui aspire à la noblesse alors qu'il appartient à une classe en réelle ascension, ou Harpagon qui pratique, comme Pantalon, une usure dépassée et contre-productive. C'est d'ailleurs en dépassant certaines limites et en prétendant se rire de la décadence de la haute aristocratie avec Don Juan ou de certaines confréries religieuses puissantes avec Tartuffe que Molière a connu ses célèbres déboires.

Mais là où le rire est peut-être le plus fort et cruel, c'est lorsqu'il s'exerce à l'égard des plus démunis comme les zanni. Ces derniers apparaissent alors que naît à Venise et dans quelques autres villes italiennes, selon Dario Fo, le capitalisme moderne qui s'appuie sur les banques et la création de l'actionnariat. Une forme qui ressemble fort à la mondialisation du travail et de la production se met alors en place puisque les actionnaires n'hésitent pas à impliquer leurs capitaux dans des guerres coloniales et à s'implanter dans les nombreuses possessions qui en découlent. Les produits, notamment alimentaires, qui proviennent de ces contrées le sont à un bien moindre coût puisque ces économies reposent sur l'esclavage si bien que les paysans de l'Italie du Nord, ruinés, furent contraints à un exode massif vers les grandes villes qu'ils envahissent, détruisant "l'équilibre du milieu, d'où rancune, mépris et dérision" (p. 74-75). Ils occupent alors la fonction peu enviable de «boucs émissaires", qui génère un rire ambigu et cruel car on se moque alors de l'extrême misère humaine, suscitant des lazzi célèbres comme ceux de la faim où le zanni mange ses membres, son sexe et ses tripes, mêlant scatologie et détresse à hurler de rire. On connaît ainsi le lazzi de la mouche où le petit insecte devient l'objet de la plus grande convoitise et d'un jeu sadique où la cruauté subie par l'affamé rebondit sur le minuscule animal. On fait dans de telles situations comiques l'expérience de ce rire paradoxal, irrépressible en dépit du thème représenté. On peut supposer qu'à l'époque même de création de ces lazzi, le public populaire riait sans arrière-pensée de ces balourds plus infortunés que lui.

Le rire provoqué par le duo de clowns composé du dominant autoritaire, le clown blanc, et du dominé, le Paillasse ou l'Auguste, est du même ordre. 
On a là un condensé des rapports de forces sociaux. On retrouve d'ailleurs le thème obsédant de la faim, du manque, de la frustration. Léternel dominé, pour survivre, doit en général, pratiquer une stratégie de «l'arrangement» qui consiste à "faire avec", à composer avec la réalité oppressive, ce qui donne lieu à des solutions fantaisistes et désopilantes où l'Auguste sauve sa dignité ou satisfait son manque, de façon comique ou poétique. Mais, selon Dario Fo:

Ce schéma fondamental, qui est celui de l'«arrangement», en recouvre un plus cruel, celui de la lutte pour la vie, où affleure souvent un cynisme destructeur de toutes les valeurs morales reçues: honnêteté, respect humain, fidélité. (p. 242)

On constate donc à quel point un tel rire peut être paradoxal, voire dilemmatique, pour le spectateur et qu'il le pousse, en quelque sorte, dans ses propres contradictions, suscitant un sentiment trouble où le sadisme le dispute à la compassion.

Les vertus euphorisantes du rire ne sont guère contestables et celles-ci, dans le contexte de réception collective du spectacle théâtral, sont renforcées par le sentiment du partage et de l'appartenance à une même communauté. En effet, si l'expérience de la tristesse, de l'angoisse pour les spectateurs relève plutôt de l'individuel et de l'intime - que de larmes vite effacées dans le noir de la salle, lorsqu'on observe discrètement les réactions du public à une scène touchante - celle du rire se communique, s'étend, se développe sans retenue. Et pourtant, on a pu constater, grâce aux riches expérimentations et réflexions de Dario Fo, à quel point le rire pouvait être complexe, paradoxal, dilemmatique même. Le rire n'est pas en soi positif ou négatif, éveilleur de conscience ou substitut naturel aux antidépresseurs; il peut être tout cela ou privilégier certains aspects selon le propos de son orchestrateur. Quand celuici est Dario Fo, on sait que tout en s'appuyant sur les pratiques traditionnelles les plus anciennes et éprouvées, il visera certainement à démasquer les leurres de la société contemporaine, inscrivant son projet théâtral dans une perspective résolument politique. Pour Vitez aussi, «l'acteur de l'ère scientifique est un acteur gai. Il sait ce qu' il fait ${ }^{\mathrm{II}}{ }^{\prime}$. Le gai savoir de l'acteur peut être lu et compris par le lecteur comme une "pédagogie» et une "politique» du rire qui contribuerait à l'accomplissement de l'homo ridens.

II. A. Vitez, L'École, op. cit., p. 49. 